\begin{tabular}{|l|c|c|c|r|}
\hline $\begin{array}{l}\text { Cuadernos de Investigación Geográfica } \\
\text { Geographical Research Letters }\end{array}$ & 2017 & No $43(1)$ & pp. 269-291 & $\begin{array}{r}\text { ISSN 0211-6820 } \\
\text { eISSN 1697-9540 }\end{array}$ \\
\hline
\end{tabular}

\title{
INFLUENCIA DE LA GLACIACIÓN EN LA RED DE DRENAJE: EL EJEMPLO DE LOS VALLES DE RESPINA Y REBUENO (ALTO PORMA, CORDILLERA CANTÁBRICA, NOROESTE DE ESPAÑA)
}

\author{
P.J. DANIS-ÁLVAREZ, J. SANTOS-GONZÁLEZ*
}

Dpto. de Geografía y Geología, Universidad de León, Campus de Vegazana, s/n, 24071 León, España.

\begin{abstract}
RESUMEN. En el presente trabajo se aborda el estudio de dos valles en la cuenca del río Porma, en la vertiente meridional de la Cordillera Cantábrica, norte de España. El primero de ellos, el de Respina, presenta múltiples evidencias glaciares, como circos, morrenas, cubetas, till o bloques erráticos que demuestran la importancia que tuvo la glaciación en ese valle. El segundo, el de Rebueno, apenas cuenta con restos glaciares y están restringidos a la zona de cabecera. La comparación de los perfiles longitudinales y transversales de ambos valles, pone de manifiesto la importancia que tuvo la glaciación en la configuración del relieve actual del valle de Respina, contrastando con las formas fluviales generadas en el valle de Rebueno. En ambos se dieron procesos distintos que configuraron un relieve con importantes diferencias a pesar de su proximidad y similares características. Además, el trabajo aborda la influencia que la glaciación ha tenido en la configuración actual de la red de drenaje, aspecto que apenas ha sido analizado previamente, y que en este caso se concreta en el desvío del río Respina y la creación de un nuevo valle, como consecuencia de una potente acumulación morrénica procedente de la lengua glaciar del valle de Silván. Se trata de un ejemplo muy significativo de la importancia que tuvo la glaciación en la red fluvial.
\end{abstract}

Glacial influence on drainage network: Respina and Rebueno valleys (Upper Porma Basin, Cantabrian Mountains, Northwest Spain)

ABSTRACT. The study addresses two valleys in the river Porma basin, on the southern slope of the Cantabrian Mountains, Spain. In the first one, the Respina Valley, there are many glacial evidences, such as cirques, moraines, till or erratic boulders, demonstrating the importance of the glaciation in this valley. The second one, the Rebueno Valley, shows very few glacial landforms restricted to the upper section of the valley. The comparison of the longitudinal and transverse profiles of both valleys, show the great influence of glaciation in the Respina valley relief, contrasting with the fluvial landforms generated in Rebueno valley. In both, distinct geomorphological processes modulated the relief 
in such a manner that, despite their proximity, they show significant differences in landscape. In addition, the paper addresses the influence of glaciation on the current configuration of the drainage network, which has scarcely been discussed previously. The main changes in drainage network are the generation of a new valley as a result of a big moraine from Silván valley that deviated the Respina river to the south. This is a very significant example of the importance of the glaciation in river network configuration in the Cantabrian Mountains.

Palabras clave: glaciarismo, Cordillera Cantábrica, valle del Porma, cambios en la red de drenaje.

Key words: glacial geomorphology, Cantabrian Mountains, Porma valley, drainage network changes.

Recibido: 3 de mayo de 2016

Aceptado: 25 de Julio de 2016

* Correspondencia: Javier Santos-González, Departamento de Geografía y Geología, Universidad de León, Campus de Vegazana, s/n, 24071 León, España. E-mail: jsango@unileon.es

\section{Introducción}

El relieve terrestre está configurado por diferentes procesos que generan formas que se superponen y modifican las ya existentes, ayudando a definir el relieve actual (Redondo Vega et al., 2013; García-Ruiz, 2015). Los glaciares son uno de los agentes de modelado más importantes de la Tierra, y sus formas, tanto erosivas como deposicionales, caracterizan el relieve de amplias superficies del planeta (Benn y Evans, 2010). En las áreas de montaña, los glaciares tienden a ocupar los valles fluviales previos, modificando su morfología; así, los perfiles de los valles glaciares suelen ser diferentes a los que tienen un origen únicamente fluvial, tanto longitudinalmente (Brocklehurst y Whipple, 2006; MacGregor et al., 2009) como transversalmente (Li et al., 2001). Además, los glaciares movilizan mayor cantidad de sedimentos que los ríos, evidenciando su mayor capacidad erosiva en zonas de montaña (Montgomery, 2002; Brook et al., 2006).

Una vez que los glaciares se retiran, las formas glaciares van siendo destruidas por otros procesos no glaciares (Church y Ryder, 1972; Ballantyne, 2002; Brardinoni y Hassan, 2006). La influencia de la glaciación se hace patente incluso mucho tiempo después en la dinámica fluvial (Hobley et al., 2010; Collins y Montgomery, 2011), produciéndose reajustes en la red de drenaje como consecuencia de la desaparición de formas de relieve dejadas por la acción glaciar (Passmore y Waddington, 2009).

Si bien son bastante numerosos los estudios sobre glaciarismo en la Península Ibérica, la influencia que la acción glaciar pudo tener en la configuración de la red de drenaje apenas ha sido analizada en detalle, con alguna excepción como los análisis de la relación entre las terrazas fluviales y depósitos glaciares (Turú y Peña, 2006; Lewis et al., 2009) o el aporte de sedimentos glaciares a conos aluviales (Gómez Villar, 1996). 
En la Cordillera Cantábrica apenas se ha abordado el estudio de la influencia que la glaciación pudo tener en la red fluvial (Jiménez Sánchez, 1999), ya sea en relación a las terrazas fluviales (González Gutiérrez, 2002; Ruiz Fernández y Poblete Piedrabuena, 2011), el aporte de sedimentos (Pérez Alberti y Valcárcel Díaz, 2001) o en reajustes en la red de drenaje (Alonso, 1998). Por ello, en este trabajo, se realiza un análisis comparado de dos valles muy próximos (Respina y Rebueno) situados en la cuenca del río Porma (vertiente sur de la Cordillera Cantábrica), con los objetivos de:

a) Realizar una descripción detallada de las formas de relieve existentes en los valles de Respina y Rebueno.

b) Comparar las morfologías existentes entre un valle glaciado y otro con una influencia muy limitada de la glaciación.

c) Analizar reajustes en la red fluvial como consecuencia de la glaciación.

\section{2. Área de estudio}

La Cordillera Cantábrica es una de las principales cadenas montañosas de la Península Ibérica con más de $400 \mathrm{~km}$ de longitud, extendiéndose de Este a Oeste en el Norte peninsular (Fig. 1). Alcanza cotas superiores a los 2000 metros en numerosos puntos y puntualmente a $2500 \mathrm{~m}$ en los Picos de Europa y en el macizo de Peña Prieta. El sector estudiado se enmarca en la vertiente sur de la Cordillera Cantábrica, en la cabecera del río Porma (cuenca del Duero, subcuenca del Esla).

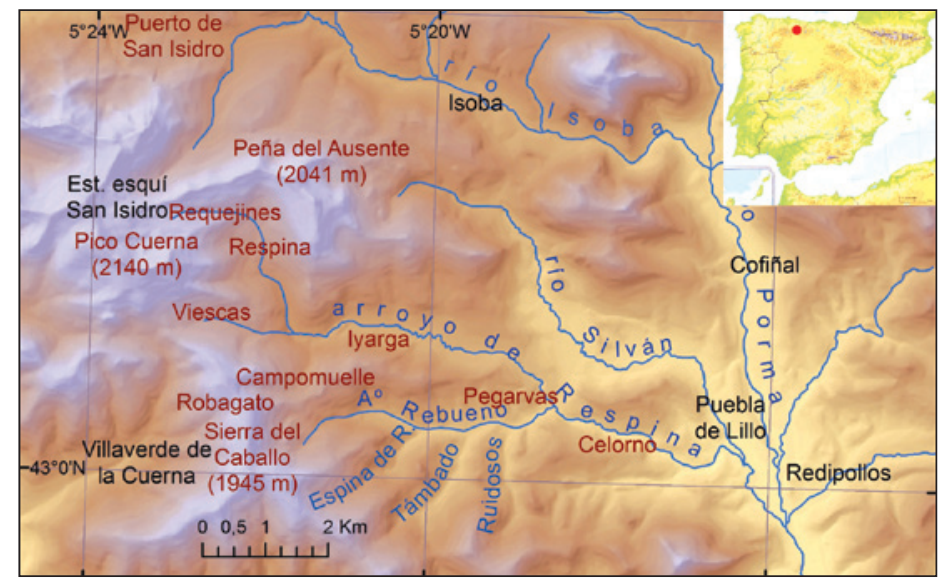

Figura 1. Localización del área de estudio, en el norte de la Península Ibérica.

El área de estudio presenta un clima oceánico de montaña, con cierta influencia mediterránea, más marcada cuando más al sur. La precipitación media anual supera los 1400 mm en Puebla de Lillo y probablemente los 1700-1800 mm en las cotas más altas (AEMET, 2011). Desde un punto de vista geológico se encuentra dentro de la Zona 
Cantábrica del Macizo Ibérico (Lotze, 1945; Julivert et al., 1972) dentro de la Unidad Bodón-Ponga, según la subdivisión más reciente propuesta por Alonso et al. (2009), que engloba las antiguas unidades del Manto del Ponga y la Cuenca Carbonífera Central (Julivert, 1965).

La sucesión estratigráfica consta de una serie inferior preorogénica correspondiente al Cámbrico Inferior representada en la Unidad de Ponga a la que se superponen materiales carboníferos integrados por lutitas, margas y calizas con la Cuenca Carbonífera Central depositados contemporáneamente con la Orogenia Varisca (sinorogénicos). Entre ambas existe una laguna estratigráfica en cuanto a materiales paleozoicos de parte del Ordovícico, Silúrico y Devónico Inferior (Álvarez Marrón y Pérez Estaún, 1988). Estas estructuras hercínicas fueron rejuvenecidas durante la Orogenia Alpina, dando lugar a la configuración del relieve actual (Alonso et al., 1996).

En concreto, se analiza el valle de Respina y su principal afluente, el valle de Rebueno. El primero cuenta con una longitud de $12 \mathrm{~km}$, entre su cabecera, enmarcada por la Peña Agujas (2141 m), el Pico Cuerna (2140 m), el Pico Redondo (2129 m), la Peña el Ausente (2040 m) y Peña de Requejines (2026 m) y su desembocadura en el río Silván, a $1128 \mathrm{~m}$, muy cerca ya del río Porma. Este valle presenta tres zonas con morfologías y litologías diferenciadas:

- Sector alto. Por encima de los 1500 m, comprende el circo de Requejines y el área ocupada por la mina de talco de Respina (abandonada). El río Respina procedente del circo de Requejines, discurre sobre la línea de un cabalgamiento entre escamas de cuarcita de la formación de Barrios con dirección E-O. A partir de los $1600 \mathrm{~m}$, aguas abajo, hay un brusco cambio de dirección, que pasa a ser N-S durante $1 \mathrm{~km}$, siguiendo la línea de cabalgamiento anterior en el interior de un anticlinal, sobre materiales del Carbonífero Superior como las calizas laminadas de la Fm. Barcaliente y las calizas y areniscas del paquete de Fresnedo.

- Sector medio. Situado entre los 1500 m (a los pies de la mina de talco) y los 1209 m correspondiendo con la confluencia de los arroyos de Respina y Rebueno. A esta área se le denomina valle de Iyarga, y en él dicho arroyo discurre sobre la línea de falla E-O que separa los materiales pertenecientes al Grupo Lena (con calizas, areniscas, carbón y lutitas) y el paquete de Fresnedo con lutitas, todo ello perteneciente al Carbonífero Superior. Presenta una morfología suave, de poca pendiente y un perfil transversal marcadamente en $U$.

- Sector bajo. Desde los 1209 m hasta los 1128 m en la confluencia del río Respina con el río Silván. Este tramo recibe el nombre de valle de Celorno. Aquí el valle presenta un perfil angosto, en forma de $\mathrm{V}$ (salvo su zona final), en este caso sin obedecer a ninguna línea de falla y siguiendo un contacto entre calizas del grupo de Lena al $\mathrm{N}$ y areniscas y lutitas también del grupo de Lena, enmarcado dentro del Manto de la Viana de la Cuenca Carbonífera Central.

Por su parte, el arroyo de Rebueno tiene una longitud de $5.1 \mathrm{~km}$, entre Sierra el Caballo (1945 m) y su desembocadura en el río Respina, a 1209 m. En ese tramo recibe 
tres afluentes de entre 2 y 2.6 km de longitud: Espina de Rebueno, Támbado y Ruidosos. Tanto el valle de Rebueno como sus afluentes presentan perfiles angostos, en forma de $\mathrm{V}$, con fondos de valle similares al tramo inferior del valle de Respina y netamente diferentes a la zona alta y media de ese valle.

\section{Estudios sobre glaciarismo en el Alto Porma}

Los estudios acerca de la importancia de la glaciación en la Cordillera Cantábrica son relativamente numerosos, remontándose en algunos casos al siglo XIX (De Prado, 1852; Saint-Saud, 1893). Entre los trabajos más recientes, se pueden destacar, entre otros, algunos análisis sobre circos (Ruiz-Fernández et al., 2009; Gómez-Villar et al., 2015), sobre depósitos glaciares (Santos-González et al., 2013b), la altitud de la línea de equilibro glaciar (Serrano Cañadas y González Trueba, 2004; Santos-González et al., 2013a) o sobre la cronología glaciar (García-Ruiz et al., 2010; Jalut et al., 2010; Frochoso et al., 2013; Jiménez-Sánchez et al., 2013; Moreno et al., 2010; Serrano et al., 2013; 2015; Rodríguez-Rodríguez et al., 2015; Ruiz-Fernández et al., 2016). A pesar de esos esfuerzos, lo cierto es que aún quedan muchos valles que apenas han sido analizados en detalle y en otros muchos no se conocen con precisión los límites que alcanzó la glaciación. Además, otros aspectos, como la relación de la glaciación con la dinámica fluvial, apenas han recibido atención.

En cuanto al área de estudio, Stickel (1929) fue el primer autor que describió con cierto detalle la morfología glaciar del Alto Porma, sugiriendo ya la ocupación de un glaciar hasta Puebla de Lillo, a 1245 m. Hasta finales de los años 80 del pasado siglo no aparecen trabajos más detallados acerca de la geomorfología glaciar de esta parte de la Cordillera Cantábrica. Así, Castañón Álvarez (1989) describió un conjunto morrénico en la confluencia de los arroyos de Respina y Rebueno, y realiza estudios de granulometría y desgaste de la fracción gruesa y morfoscopía de las arenas de la terraza fluvioglaciar situada en la confluencia del valle de Respina con el del Porma. Asimismo incluyó una descripción de los depósitos de sedimentos glaciares, fluvioglaciares y torrenciales en la confluencia del arroyo de Rebueno con el río Respina. Por su parte, Álvarez Marrón et al. (1990), representaron algunas de las principales formas glaciares en la cartografía geomorfológica a escala 1:50.000 para la hoja 79 (Puebla de Lillo) del mapa geológico del IGME.

Rodríguez Pérez (1995) realizó un estudio geomorfológico de la cabecera del Porma y describió algunas de las principales formas glaciares en la vertiente $\mathrm{N}$ de la sierra de Sentiles, cabecera donde se desarrolló el aparato glaciar del que el sistema de Respina era tributario. Por otro lado, Frochoso Sánchez y Castañón Álvarez (1998) mencionaron las morrenas bien definidas que separan los valles de Respina y Silván.

Por su parte, Alonso Herrero (2002), llevó a cabo un estudio de la glaciación en los valles del Esla y Porma e hizo una breve descripción del aparato glaciar de Respina así como de las diferentes morfologías de origen glaciar, como la morrena lateral y la laguna de obturación en la margen izquierda del arroyo de las Viescas. En el año 2007, Redondo Vega (com. personal) observó algunos restos de interés aguas abajo de Puebla de Lillo, destacando un bloque errático de cuarcita de 3.6 × 3.5 x $8.3 \mathrm{~m}$ que pone en evidencia la 
existencia de un máximo glaciar anterior a la fase en la que se depositaron las morrenas de esa localidad. Dicho bloque es incluido en los Lugares de Interés Geológico de la Provincia de León (Fernández Martínez et al., 2009). Posteriormente, Redondo Vega et al. (2014) describieron los restos glaciares más bajos del glaciar del Porma, situando el frente a sólo 1100 m de altitud, y Danis Álvarez (2014), realizó un exhaustivo trabajo de campo en los valles de Respina y Rebueno, reconociendo las principales formas glaciares de estos valles, lo que sirve de base para el presente estudio.

Más recientemente, Rodríguez-Rodríguez et al.(2016), a partir de una tesis doctoral no publicada (Rodríguez Rodríguez,2015), realizaron una reconstrucción geomorfológica de la dinámica glaciar del valle de Porma, estimando la Línea de Equilibro Glaciar en unos $1575 \mathrm{~m}$ y llevando a cabo dataciones con Berilio 10. Según estos autores existió un máximo glaciar antiguo $(113.9 \pm 7.1 \mathrm{ka})$, con avances en $55.7 \pm 4$ ka y una extensión menor durante el Último Máximo Glaciar global.

\section{Metodología}

La elaboración de este estudio se ha basado en el reconocimiento de las formas de relieve a partir de, en primer lugar, la recopilación de la bibliografía previa, la fotografía aérea, la cartografía topográfica (hojas 79-III y 79-IV) y la observación de los modelos digitales de terreno (LIDAR) y, en segundo lugar, del trabajo de campo, lo que ha permitido la elaboración de cartografía geomorfológica. Ésta se ha realizado utilizando el programa ArcGIS 10.3.

El trabajo de campo, centrado en primavera y verano de 2014, ha sido intensivo en todo el área de estudio y en especial en las áreas marginales y en la confluencia de los arroyos de Rebueno y Respina, para poder estimar con precisión la dinámica glaciar y postglaciar del valle. Estas salidas de campo han sido imprescindibles para la identificación de las diferentes formas de erosión y sedimentación, así como la modificación de cauces en el área de estudio, aportando de esta manera la información necesaria para analizar las interrelaciones glaciarismo-red de drenaje.

A partir de la cartografía topográfica citada anteriormente y los modelos digitales del terreno, se han podido elaborar diferentes perfiles trasversales utilizando el programa Surface 10 y longitudinales de detalle en los sectores más representativos dentro del área de estudio.

En el valle de Rebueno, a partir de esta reconstrucción de la masa glaciar se ha calculado la línea de equilibro glaciar (LEG) o Equilibrium Line Altitude (ELA), para lo cual se ha utilizado el método AABR (Area Altitude Balance Ratios) desarrollado por Furbish and Andrews (1984) y Benn y Gemmell (1997), que es el más utilizado actualmente. Calculada la superficie en cada franja altitudinal en las que se ha dividido la lengua glaciar, se ha estimado la LEG mediante la hoja de cálculo del programa Excel diseñada por Osmaston (2005). Para realizar los cálculos hemos utilizado el rango de valores calculado por Rea (2009) para glaciares de latitudes medias actualmente, que se sitúa en $1.9 \pm 0.81$. 


\section{Resultados}

\subsection{Formas glaciares en el Valle de Respina}

Las formas de origen glaciar son muy abundantes a lo largo de casi todo el valle, tanto erosivas como deposicionales:

\subsubsection{Formas glaciares erosivas}

En el valle de Respina existen cuatro circos glaciares bien formados (Tabla 1) entre los que destaca el de Requejines, que se desarrolla sobre cuarcitas del Ordovícico de la Fm. Barrios. Se trata de un circo compuesto, con una parte situada en la cabecera del río Isoba y otra en el de Respina. Debido a la dureza de los materiales, las formas existentes presentan un elevado estado de conservación, a pesar del impacto y degradación sufridos por las instalaciones de Estación Invernal de San Isidro y la mina a cielo abierto de Respina. El hielo de este circo sobrealimentaba otro circo emplazado a los pies de las Agujas de la Cuerna (2117 m), cuyas dimensiones son algo más modestas (Fig. 2).

Tabla 1. Datos morfométricos de los circos glaciares del valle de Respina. La superficie está medida en hectáreas y las altitudes y dimensiones de los circos en metros.

\begin{tabular}{ccccccc}
\hline Circo & Orientación & Superficie & $\begin{array}{c}\text { Altitud } \\
\text { máxima }\end{array}$ & $\begin{array}{c}\text { Altitud } \\
\text { mínima }\end{array}$ & Longitud & Anchura \\
\hline Requejines & ENE & 101 & 2140 & 1800 & 1100 & 968 \\
Agujas de la Cuerna & $\mathrm{E}$ & 42 & 2117 & 1649 & 952 & 629 \\
Rabogato & $\mathrm{NE}$ & 20 & 1907 & 1668 & 515 & 480 \\
Peña Ausente & $\mathrm{SE}$ & 35 & 2041 & 1770 & 690 & 500 \\
\hline
\end{tabular}

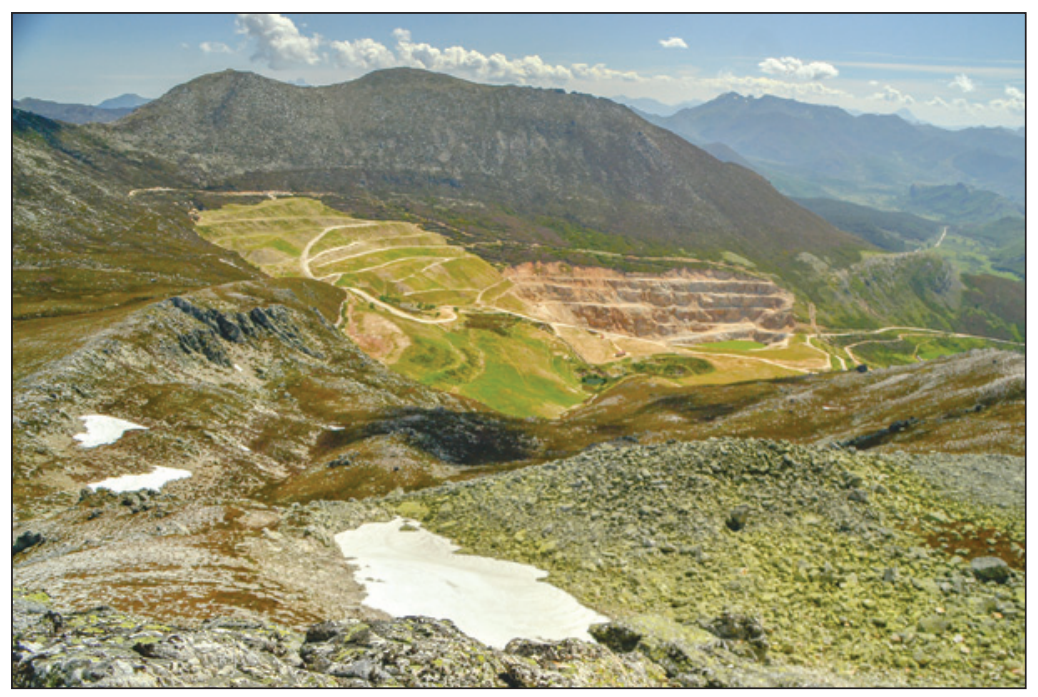

Figura 2. Circo glaciar bajo el Pico Redondo. Se puede observar también, en primer plano, el frente de un pequeño glaciar rocoso relicto. En la parte central la mina de talco de Respina. 
Los otros dos circos alcanzan un menor desarrollo. El primero de ellos se encuentra en la Loma de Rabogato, con orientación NE y una superficie de 20 ha. El segundo se ubica en la vertiente Sur de la Peña Ausente y es algo mayor, alcanzando las 35 ha. Además, también había zonas de acumulación de hielo dónde no se generaron verdaderos circos, como en la vertiente meridional del Pico Redondo. Las dimensiones tan reducidas de estas últimas formas pueden relacionarse con la orientación hacia el Sur, muy poco favorable para el desarrollo glaciar debido al barrido de la nieve de las laderas a barlovento (laderas orientadas al oeste) y a la elevada insolación (laderas orientadas al sur).

Tras su nacimiento en el circo de Requejines, el valle de Respina presenta nítida forma de artesa glaciar, con alternancia de umbrales y zonas sobreexcavadas, siguiendo la dirección de las líneas de falla sobre la Fm. Barrios. Tras un brusco cambio de dirección y de pendiente, en su tramo medio (Iyarga), el valle presenta una morfología típica de valle glaciar con una sección trasversal en U. Es en este tramo donde los valles afluentes de Viescas (Fig. 3) y Campomuelle aparecen colgados $150 \mathrm{~m}$ sobre el fondo de valle principal, salvando ese desnivel los respectivos arroyos mediante cascadas. Por su parte, el propio valle de Respina, aparece suspendido unos $110 \mathrm{~m}$ sobre el valle de Silván, por donde fluía gran parte del hielo procedente de la muy extensa área de acumulación del puerto de San Isidro, al norte de la zona de estudio. Estos desniveles están relacionados con la distinta competencia de los glaciares, lo que permite inferir la mayor potencia de hielo del valle de Silván respecto al de Respina, y del valle de Respina respecto a sus afluentes.

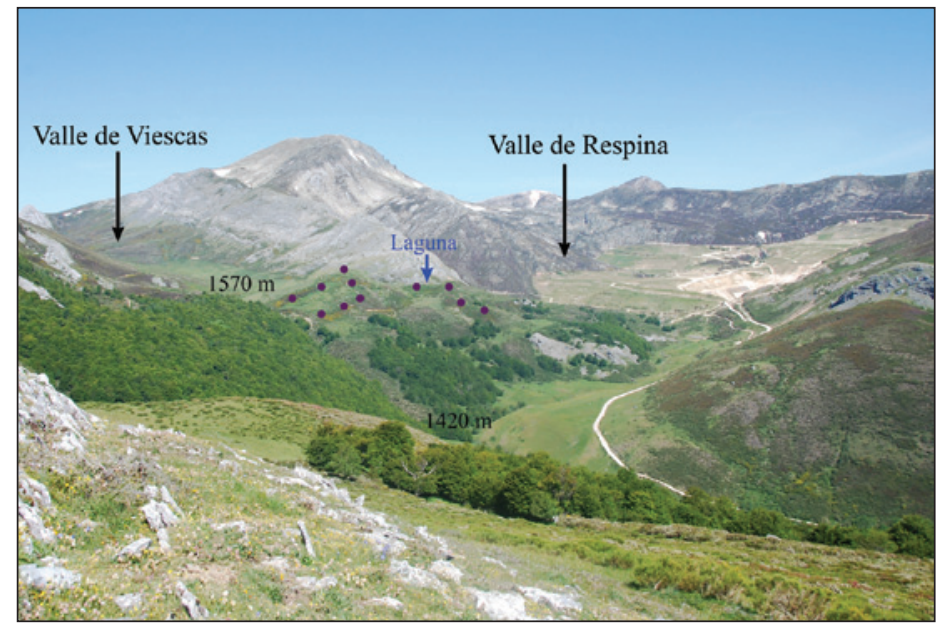

Figura 3. Vista de la cabecera del valle de Respina y la mina de talco. Se puede apreciar que el valle afluente de Viescas, dónde se generó una pequeña lengua glaciar, aparece suspendido 150 metros sobre el valle de Respina, donde la potencia de hielo fue mucho mayor gracias a las mejores condiciones para la acumulación de hielo. Los puntos representan la localización de varias morrenas. 
El tramo bajo del valle, conocido como Celorno, incluye el sector comprendido entre la confluencia con el arroyo de Rebueno (donde existe un umbral glaciar) y la desembocadura del río Respina en el río Silván, muy cerca de Puebla de Lillo. Se desarrolla sobre materiales deleznables del Carbonífero superior como calizas y lutitas. Este valle se caracteriza por su perfil en V, su angostura y el fuerte encajamiento que presenta frente a otros valles próximos desarrollados sobre los mismos materiales y que cuentan con perfiles mucho más suaves. En este tramo no se observan restos glaciares salvo en su parte inicial.

\subsubsection{Restos glaciares deposicionales}

Los sedimentos depositados por la lengua glaciar que discurrió a lo largo del valle de Respina, desde su cabecera en Requejines hasta su confluencia con la lengua de hielo del valle del Silván, se presentan en forma de diferentes acumulaciones morrénicas.

En la parte alta (circo de Requejines) las morrenas son de reducidas dimensiones y aparecen junto a algunos glaciares rocosos (Gómez Villar et al., 2011). Estas morrenas, junto a las pequeñas cubetas de sobre-excavación, han generado pequeñas lagunas de escasa profundidad, la mayor de las cuales tiene 0.63 ha. Más destacado es el conjunto de morrenas laterales en la confluencia entre los arroyos de Viescas y Respina en muy buen estado de conservación y con tres arcos bien definidos. Una de las morrenas ha generado una pequeña laguna yuxtaglaciar (0.17 ha) que ha sido catalogada como Lugar de Interés Geológico de la Provincia de León (Fernández Martínez et al. 2009). También en el valle de Viescas aparecen al menos tres arcos morrénicos (Fig. 4A), lagunas y turberas intramorrénicas, que indican en este caso espesores de hielo de más de $200 \mathrm{~m}$ de la lengua glaciar.Además, a lo largo del valle del Iyarga en su margen $\mathrm{N}$ se puede apreciar una morrena lateral que indica un espesor del hielo de $170 \mathrm{~m}$ y que es responsable de la formación de la laguna de obturación de Robledo (1.6 ha) (Fig. 4D), suspendida sobre el valle principal en su margen izquierda. También en la margen izquierda del valle, existe un conjunto de bloques erráticos de cuarcita de gran tamaño (5 m x 4 m como máximo) correspondientes a la Fm. Barrio (Fig. 4C), que han resistido la erosión. Destacan los existentes sobre las calizas de la Fm. Barcaliente que aparecen en el flanco $\mathrm{N}$ del valle de Iyarga a unos $160 \mathrm{~m}$ respecto al fondo del valle y que corresponden al máximo espesor de hielo.

Pero el conjunto morrénico más destacado del área de estudio aparece en el sector final de este tramo del valle, donde se desarrollan tres morrenas de grandes dimensiones entre los valles de Silván y Respina. En este caso evidencian unos espesores cercanos a los $100 \mathrm{~m}$ por parte de la masa de hielo de Respina y de $150 \mathrm{~m}$ en la fase de máximo por parte de la lengua glaciar del Silván. La confluencia de ambas masas de hielo y, por consiguiente, de sus morrenas laterales ha originado una importante acumulación morrénica próxima a los $120 \mathrm{~m}$ de espesor. Este conjunto de morrenas, supera los 2.3 $\mathrm{km}$ de longitud e incluye arcos morrénicos menores, como el de la laguna de Agüezo, de 0.18 ha, situada al inicio del valle de Celorno y cerrada por una morrena frontal, así como restos de till y una morrena lateral en la confluencia de Rebueno. Debido a la densa cubierta vegetal, las secciones de till son escasas, pero se puede ver la estructura interna del depósito en algunos puntos (Fig. 4B), mostrando la diversidad litológica del depósito y la presencia de numerosos cantos estriados. 


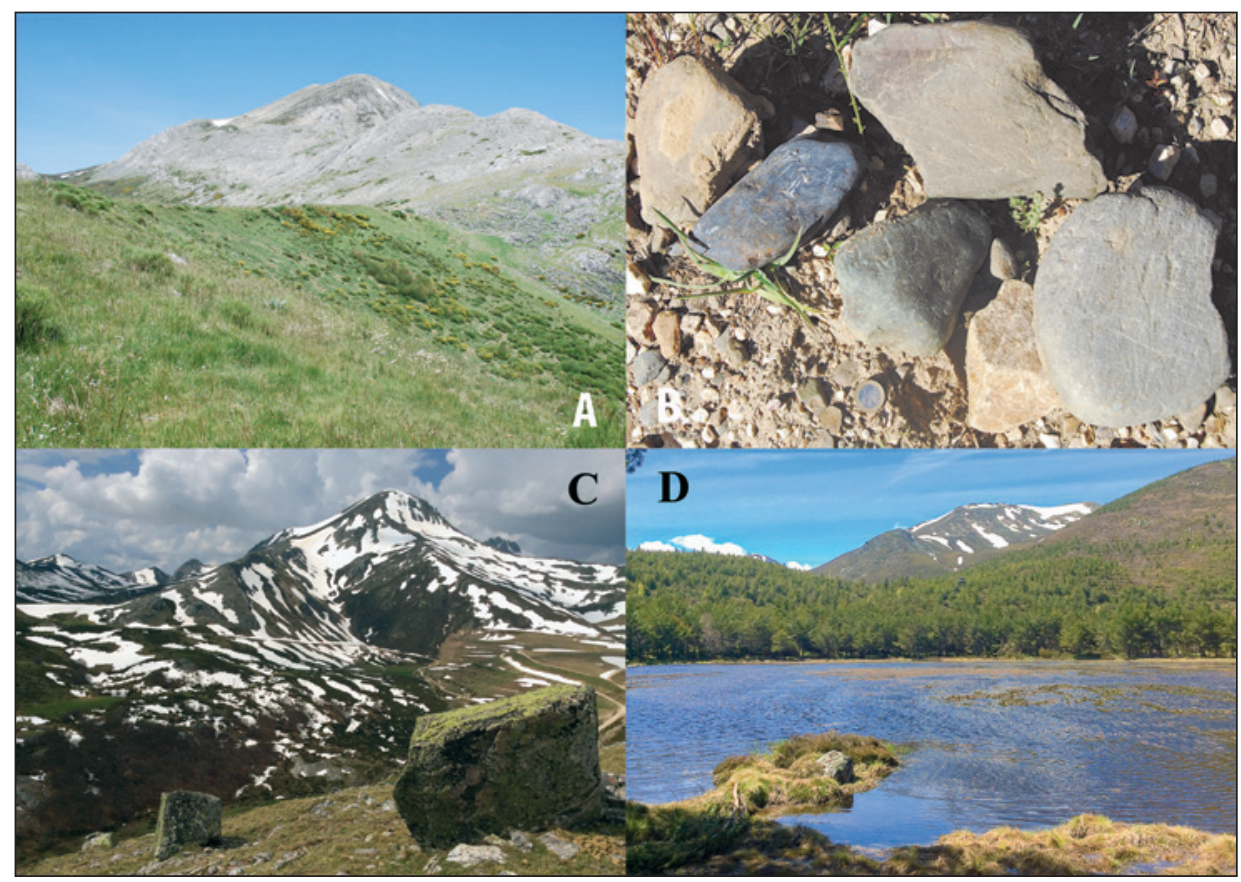

Figura 4. A) Detalle de la morrena lateral derecha del valle de Respina, en el entorno de la Majada de Valporquero. Esta morrena obturó el valle de Viescas generando un paleolago. Al fondo, la vertiente sur del Pico Redondo $(2129 \mathrm{~m})$. B) Detalle de algunos cantos estriados en la morrena lateral situada entre los valles de Silván y Respina. La diversidad litológica del depósito es elevada, y evidencia el arrastre de materiales a lo largo de todo el valle. Una moneda de 1 euro como escala. C) Bloques erráticos de cuarcitas de la Formación Barrios sobre un umbral de calizas de la Fm. Barcaliente. Los bloques se encuentran a $160 \mathrm{~m}$ sobre el fondo de valle actual, en el tramo alto del valle de Respina. Al fondo el Pico Redondo (2129 m).

D) Laguna de Robledo, en segundo plano, circo glaciar bajo la cima del Pico Ausente.

Por último, tienen interés por su localización los boques erráticos que se encuentran en el entorno de la confluencia del arroyo de Rebueno y el río Respina, en la zona recreativa de Pegarúas y que indican que el valle de Rebueno debió quedar obturado, al menos durante algún tiempo, por el glaciar de Respina.

\subsection{La morfología del Valle de Rebueno}

Contrastando con el rosario de restos glaciares existentes en el valle de Respina, el valle de Rebueno presenta una morfología netamente diferenciada, a pesar de la proximidad de ambos valles y de unas dimensiones comparables (hasta su confluencia el valle de Respina cuenta con 1490 ha por 1170 ha de Rebueno).

Si analizamos las cabeceras, ocupadas por circos bien desarrollados en el valle de Respina, aparecen aquí con circos poco definidos en los que apenas existen zonas sobreexcavadas. De hecho, solo en el valle principal de Rebueno se puede hablar de un 
verdadero circo, de $550 \mathrm{~m}$ de anchura por $330 \mathrm{~m}$ de longitud, una altitud máxima de $1945 \mathrm{~m}$ y una altitud mínima de $1770 \mathrm{~m}$. A la salida del mismo son visibles un par de morrenas laterales, de diferentes fases, pero que no descienden de los $1350 \mathrm{~m}$ de altitud. La riqueza de lagunas glaciares del valle de Respina contrasta también con la total ausencia de las mismas en el valle de Rebueno, debido a que no hay cubetas de sobreexcavación destacadas ni morrenas capaces de cerrar el drenaje o condicionarlo.

Las reducidas dimensiones del glaciarismo en el valle de Rebueno (Fig. 5) permiten calcular con cierta facilidad la Línea de Equilibro Glaciar (LEG) en este valle. Por el contrario, el glaciar de Respina estuvo conectado con el resto del glaciar del Porma, por lo que la LEG debe estimarse de forma conjunta para todo el aparato, como realizaron Rodríguez-Rodríguez et al. (2016). Así, aplicando el método AABR, la LEG en el valle de Rebueno estaría situada a $1674 \pm 25$ m. Este valor es unos 100 metros más elevado que el calculado por Rodríguez-Rodríguez et al. (2016) para el complejo glaciar del Porma (1570 m), lo cual se corresponde con la posición meridional del valle de Rebueno respecto a las zonas más húmedas y frías de la cabecera del Porma.

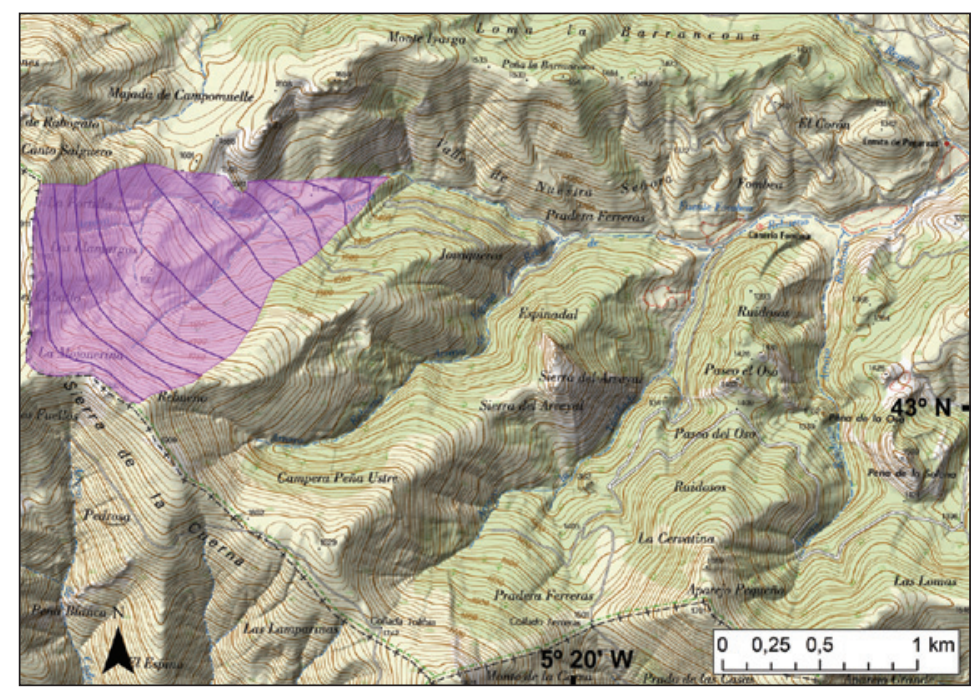

Figura 5. Reconstrucción del glaciar del valle de Rebueno a partir de las observaciones geomorfológicas. Las líneas indican las curvas de nivel del hielo durante el máximo avance. Base topográfica: IGN.

\subsection{Comparativa entre los perfiles longitudinales}

Se ha realizado una comparación entre los perfiles de los valles del ámbito de estudio (Fig. 6). A pesar de que se trata de valles con una longitud y altitud diferentes, creemos que su proximidad y sus semejantes características geológicas y litológicas, permiten realizar una comparación entre los mismos, de la que extraemos algunas conclusiones. 
Así, lo primero que llama la atención son las acusadas rupturas de pendiente que aparecen en el valle de Respina (Fig. 6). En cabecera, esas rupturas están marcadas por el final del circo glaciar de Cebolledo (Fig. 6, a), pero también por cambios en la dirección del valle, lo que se traduce en incremento de pendiente (Fig. 6, b-c). Este cambio coincide con las diferentes líneas de falla y cabalgamiento que existen en esta zona, y con un cambio de la litología del sustrato y por consiguiente de resistencia de dichos materiales que lo componen, puesto que pasan de ser cuarcitas (Fm. Barrios) a alternancias de lutitas y calizas (Grupo Lena).

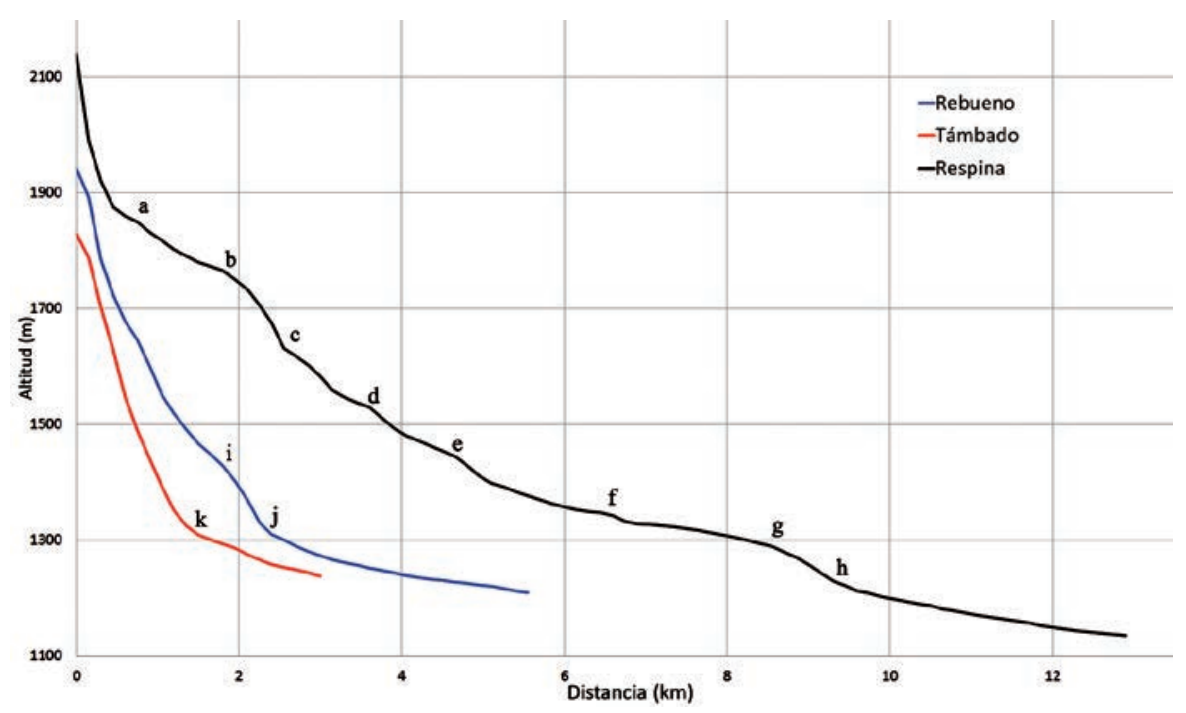

Figura 6. Perfiles longitudinales de los valles de Respina, Rebueno y Támbado.

Posteriormente, el valle presenta una morfología con pequeños umbrales glaciares (Fig. 6, d, e, f) que se alternan con cubetas parcialmente erosionadas donde la pendiente disminuye. A unos $1300 \mathrm{~m}$ de altitud, la pendiente aumenta considerablemente (Fig. 6, g) debido a que el río Respina se encaja en las morrenas laterales del valle de Silván, que debieron obturar el valle aguas arriba, como explica la escasa pendiente del valle aguas arriba de ese punto. Una vez sobrepasadas las morrenas (Fig. 6, h), en la confluencia del arroyo Rebueno, la pendiente disminuye pero, a pesar de la mayor capacidad del río, sigue siendo relativamente alta respecto a otros tramos de aguas arriba.

En el valle de Rebueno, el perfil longitudinal muestra unas rupturas de pendiente mucho menos marcadas, aunque se puede observar un tramo de menor desnivel que coincide con un pequeño rellano glaciar parcialmente desmantelado (Fig. 6, i), a partir del cual se produce un encajamiento que finaliza bruscamente con los últimos restos glaciares sobre los que el arroyo se encaja (Fig. 6, j). A partir de ese punto, el perfil no muestra rupturas de pendiente significativas, a pesar de que sigue una dirección prácticamente paralela al valle de Respina, donde sí se observan esos cambios. 
En cuanto a los pequeños afluentes del valle de Rebueno, como el de Támbado, el perfil que muestran se corresponde con el característico de morfologías fluviales, sin rupturas de pendiente nítidas, y solo se puede detectar un cambio significativo cuando el valle tiene mayor capacidad por recibir el aporte de pequeños barrancos secundarios (Fig. 6, k).

\subsection{Comparativa entre los perfiles transversales}

El valle de Respina presenta un perfil transversal netamente diferente en función del tramo considerado. Así, en su tramo medio el perfil se corresponde con una típica artesa glaciar, con un fondo de valle bastante plano enmarcado por laderas en las que se pueden observar hombreras glaciares (Fig. 7). En la margen derecha del valle una potente morrena lateral produce localmente pequeñas obturaciones, como la que ocupa la Laguna de Robledo.

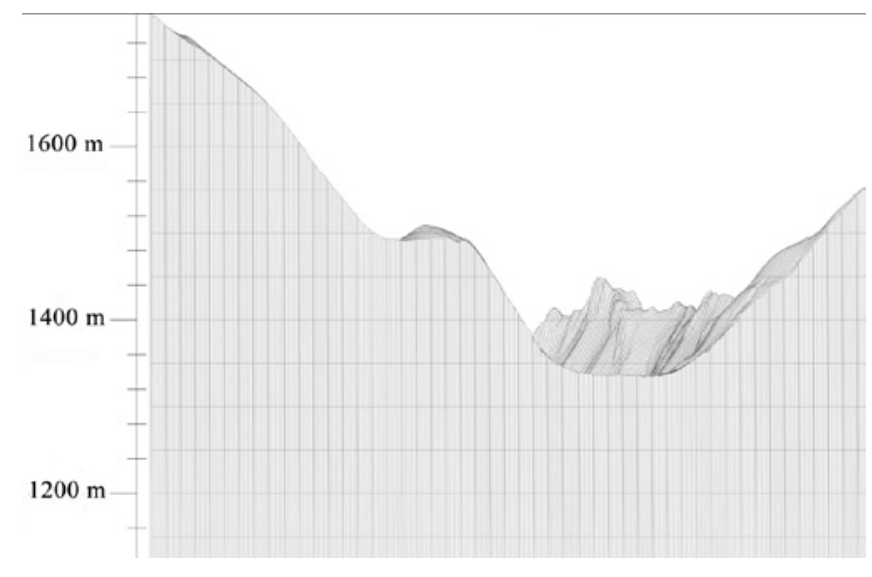

Figura 7. Perfil transversal del valle de Respina en su tramo medio.

Sin embargo, su tramo bajo (Celorno) presenta un perfil netamente diferente, con forma de $\mathrm{V}$, más encajado cerca del cauce y con una marcada ruptura en la ladera a partir de la cual se incrementa la pendiente media (Fig. 8a). Únicamente al comienzo de ese tramo existe un pequeño rellano en la ladera que coincide con una laguna cerrada por una morrena (Fig. 8b), donde hemos localizado los últimos restos glaciares en este valle. A partir de ese punto, la ruptura de pendiente desaparece. Este perfil contrasta enormemente con el que presenta el valle de Silván, que discurre paralelo inmediatamente más al norte, donde hay restos glaciares abundantes y donde existe una marcada morrena lateral en su margen izquierda (Fig. 8c).

Por su parte, el valle de Rebueno y sus afluentes presentan una morfología en V, típicamente fluvial, donde no se observan rupturas de pendiente significativas en las laderas (Fig. 9) y donde tampoco hemos apreciado ninguna evidencia de la acción glaciar. 


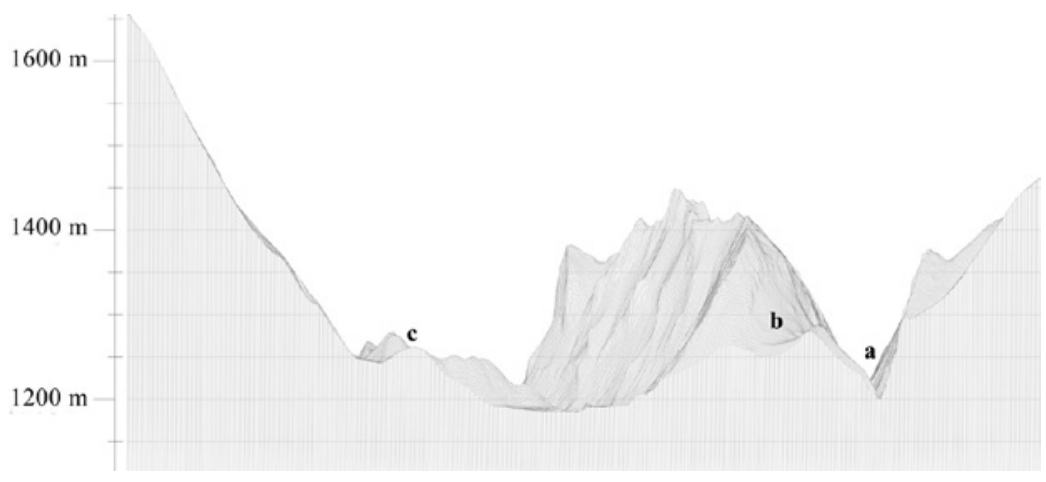

Figura 8. Perfil transversal de los valle de Respina (tramo de Celorno) y Silván.

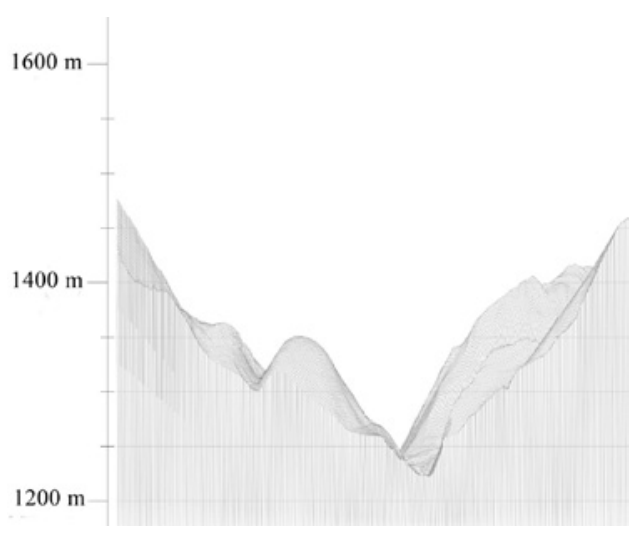

Figura 9. Perfil transversal del valle de Rebueno en su tramo medio.

\section{Discusión}

\subsection{Comparación entre valles con morfología glaciar y fluvial}

La observación detallada de las formas de relieve de los valles de Respina y Rebueno y su comparación permiten extraer algunas conclusiones relacionadas con las diferencias morfológicas en ambos valles. Así, en el valle de Respina las formas glaciares son evidentes, con la presencia de circos, morrenas, cubetas, bloques erráticos y till a lo largo de buena parte de su recorrido, hasta su confluencia con el arroyo de Rebueno. Por el contrario, en el de Rebueno las formas glaciares se limitan a un pequeño circo y alguna morrena parcialmente desmantelada en su cabecera, mientras que en sus valles afluentes apenas se pueden observar formas glaciares fuera de las zonas más elevadas.

Esa diferencia en la presencia o no de formas glaciares se traduce en la existencia de perfiles tanto transversales como longitudinales significativamente distintos en ambos casos. Así, en el valle de Respina se observa un perfil longitudinal caracterizado por la 
existencia de rupturas de pendiente que, en la mayoría de los casos, coincide con formas derivadas de la acción glaciar (umbrales, cubetas, morrenas) y un perfil transversal (en su tramo alto y medio) en forma de $\mathrm{U}$, con un fondo de valle relativamente plano y presencia de hombreras y morrenas laterales en las laderas. Por el contrario, en el valle de Rebueno las rupturas de pendiente en su perfil longitudinal son mínimas y restringidas a la cabecera del valle, y su perfil transversal tiene una marcada forma de $\mathrm{V}$, sin rupturas de pendiente significativas. También es significativo el hecho de que los pequeños barrancos laterales generan conos de dimensiones considerablemente mayores en el valle de Respina que en el valle de Rebueno, lo que parece estar relacionado con el aporte "extra" de material morrénico en el primero de ellos.

Estas observaciones ponen en evidencia que, a pesar de la proximidad de ambos valles, la acción glaciar modificó sensiblemente la morfología del valle de Respina, mientras que su acción fue muy limitada en el valle de Rebueno y restringida a la cabecera. Estas diferencias se avienen con las observaciones de Li et al. (2001), que indican que los valles que han estado glaciados suelen presentar una morfología más abierta (en forma de U) que los valles no glaciados en ambientes similares, y con las de Brocklehurst y Whipple (2006) y MacGregor et al. (2009) que demuestran que los perfiles de los valles modelados por glaciares suelen ser significativamente diferentes a los mostrados por cursos fluviales no afectados por la dinámica glaciar, que presentan un perfil más irregular, con rupturas de pendiente bien marcadas.

Todo ello contrasta con la reconstrucción glaciar presentada por RodríguezRodríguez et al. (2016), que señalan que el valle de Rebueno y sus afluentes también estuvieron ocupados por una lengua de hielo de varios kilómetros de recorrido, a pesar de que en el mapa geomorfológico no señalan la presencia de formas ni depósitos glaciares. Para estos autores, la LEG calculada para todo el conjunto del Porma era de $1570 \mathrm{~m}$. Como hemos visto, la posición meridional del valle de Rebueno debió provocar un posicionamiento de la LEG hacia los $1674 \pm 25$ m que hemos estimado en este sector. Ese incremento de la LEG según nos desplazamos hacia el sur era la norma habitual de la Cordillera Cantábrica, debido probablemente a las condiciones más secas de la vertiente meridional (Santos-González et al., 2013a). En todo caso, incluso utilizando el valor propuesto para el conjunto del Porma, y siguiendo la reconstrucción glaciar propuesta por estos autores, la superficie de acumulación $(\approx 110$ ha, 39\%) sería sensiblemente inferior a su supuesta zona de ablación ( $\approx 170$ ha, $61 \%)$, lo que no está en consonancia con la dinámica glaciar. Así, el porcentaje de zona de acumulación en glaciares templados suele estar próximo al 65\% y solo en casos excepcionales se acerca al 50\% (Rea, 2009).

Lo mismo ocurre en los pequeños valles afluentes del valle de Rebueno (Espina de Rebueno, Támbado y Ruidosos), donde estos autores señalan la existencia de pequeñas lenguas glaciares que se unían a la principal. Pues bien, por ejemplo, en el último de estos valles la superficie por encima de $1570 \mathrm{~m}$ (volvemos a incidir en que dada la posición meridional del valle, la LEG, según nuestros cálculos fue 100 metros más alta) es de sólo unas 24 ha, mientras que la supuesta zona de ablación sería de más de 60 ha (71\% del total de la superficie del glaciar). Esto contrasta con la ausencia de restos glaciares observados en estos pequeños valles, excepto en las zonas más elevadas, con una LEG 
que probablemente se situó incluso por encima de los 1800 m, dada su orientación más desfavorable respecto al valle de Rebueno, y que solo debió permitir la existencia de pequeños lentejones de hielo.

Todas las evidencias señaladas, nos llevan a considerar que el valle de Rebueno y sus afluentes permanecieron deglaciados en la mayor parte de su recorrido, y de ahí la nítida morfología en $\mathrm{V}$ que presentan, que es muy diferente a la de todos los valles próximos más septentrionales, que además sí presentan claras evidencias de la acción glaciar (morrenas laterales, till, valles en U, cubetas, obturaciones laterales, etc.). Aparte de las cabeceras, los únicos restos glaciares evidentes (también señalados por Rodríguez-Rodríguez et al., 2016) aparecen en el entorno de la ermita de Pegarúas, pero por su posición topográfica son claramente continuación de la morrena externa del valle de Respina, que debió obturar durante algún tiempo el valle de Rebueno, dando lugar una laguna.

\subsection{Influencia de la glaciación en la red de drenaje}

La actual red de drenaje presenta un dirección dominante $\mathrm{O}-\mathrm{E}$ en los valles de Iyarga y Celorno, con la única excepción de la zona de la mina de talco de Respina que se vuelve N-S. Sin embargo, numerosos indicios nos indican que la actual disposición de los cursos fluviales no se corresponde totalmente con la que tenían antes de la glaciación.

Este aspecto es especialmente evidente en el entorno de la confluencia de los valles de Silván, Respina y Rebueno. En esta zona, el río Respina sufre un brusco cambio de dirección y, en vez de desembocar directamente en el río Silván, se desvía hacia el SE, encajándose notablemente aguas abajo de la confluencia con el arroyo de Rebueno. En ese punto se inicia el conocido como valle de Celorno, más angosto que todos los del entorno.

Si observamos en detalle ese lugar, el cambio de dirección del río Respina y el incremento que presenta en su pendiente, coincide con la presencia de potentes morrenas laterales del valle de Silván, que desvían el curso del río Respina. Esas diferencias morfológicas, nos llevan a plantear la siguiente evolución del relieve (Fig. 10):

(i) En una fase previa a la glaciación, el río Respina desembocaría en el río Silván, en un lugar próximo a Los Soportales. En el tramo conocido como Celorno no existiría un valle bien definido, sino un pequeño barranco que desaguaría en el arroyo Patina.

(ii) Durante la glaciación, entre los valles de Silván y Respina se generaron potentes arcos morrénicos (Fig. 11) que impedían la evacuación del agua del glaciar de Respina, que se vio obligado a virar hacia el suroeste, erosionando la divisoria existente con el valle de Celorno.

(iii) Tras la retirada de los glaciares a cotas más altas, el río Respina se encajó en las morrenas y progresivamente las fue desmantelando, y no ha sido capaz de volver a su antiguo curso, sino que sigue desaguando por el valle de Celorno, creando un valle angosto en ese tramo. 


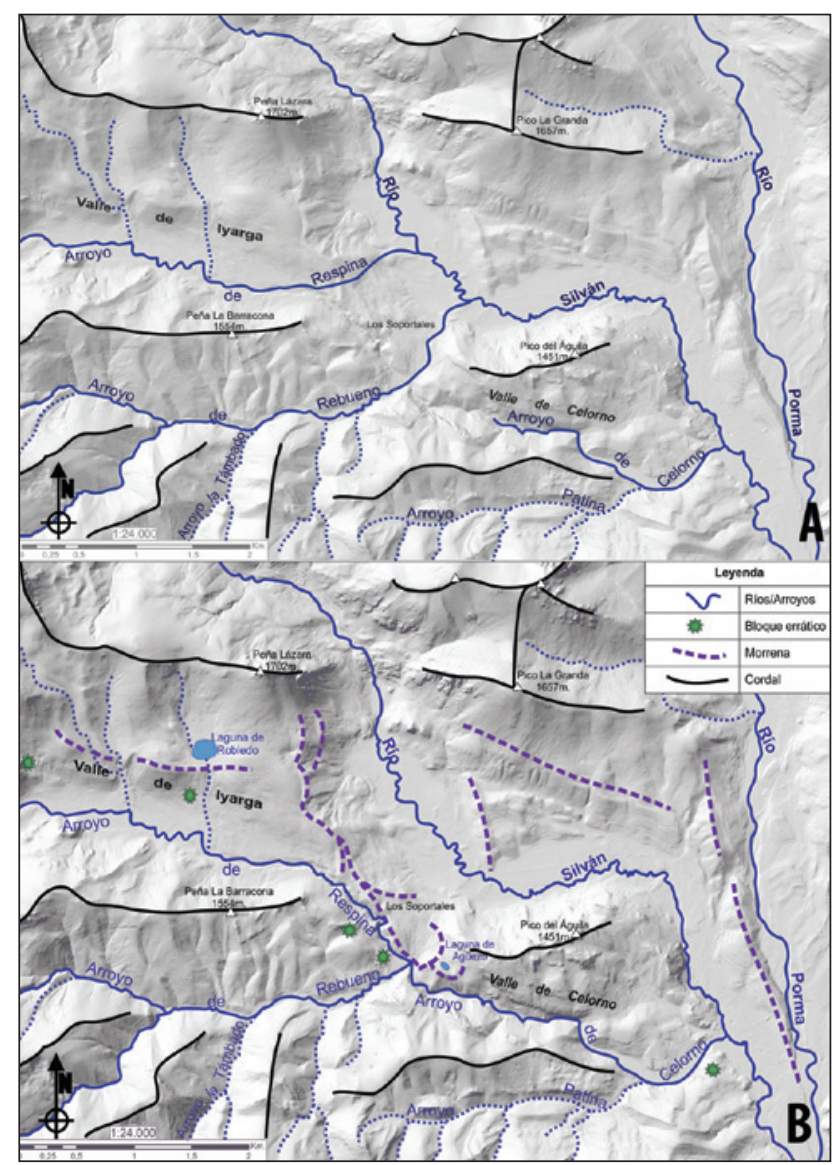

Figura 10. Esquema topográfico mostrando los cambios producidos en la red de drenaje en el área de estudio. La potente acumulación morrénica entre los valles de Respina y Silván, desvió el curso del río Respina obligándolo a desarrollar un nuevo valle (Celorno), con un trazado (b) sensiblemente diferente al original (a). Ese nuevo tramo de valle presenta una morfología angosta y muy diferente a los del entorno.

Este proceso habría supuesto la creación de un valle nuevo de más de $3 \mathrm{~km}$ de longitud, entre las morrenas del valle de Silván y Puebla de Lillo, donde se encuentra ahora la confluencia entre los ríos Respina y Silván. Se trata de un valle angosto (Fig. 12), con un encajamiento muy marcado de unos 70 metros sobre las laderas que lo flanquean y que solo a partir de la confluencia del Arroyo de la Patina presenta un perfil más tendido. Las diferencias entre los perfiles longitudinales y transversales que se han observado apoyan esta hipótesis.

Es posible que durante el máximo avance glaciar algo de hielo se canalizara por la cabecera de ese tramo, facilitando así la creación del valle, pero los restos observados se limitan al tramo superior del valle, dónde existen pequeños arcos morrénicos, no habiéndose observado restos claros en el tramo medio del mismo. 
En cuanto a los bloques existentes próximos a Puebla de Lillo y en la desembocadura del valle de Celorno, (Rodríguez-Rodríguez et al., 2016), dadas sus características y su posición altitudinal (a $1170 \mathrm{~m}$, la misma cota que la morrena central existente entre los valles del Porma y Silván a esa altura del valle), consideramos que deben relacionarse con los restos de la morrena lateral derecha del glaciar del Porma.

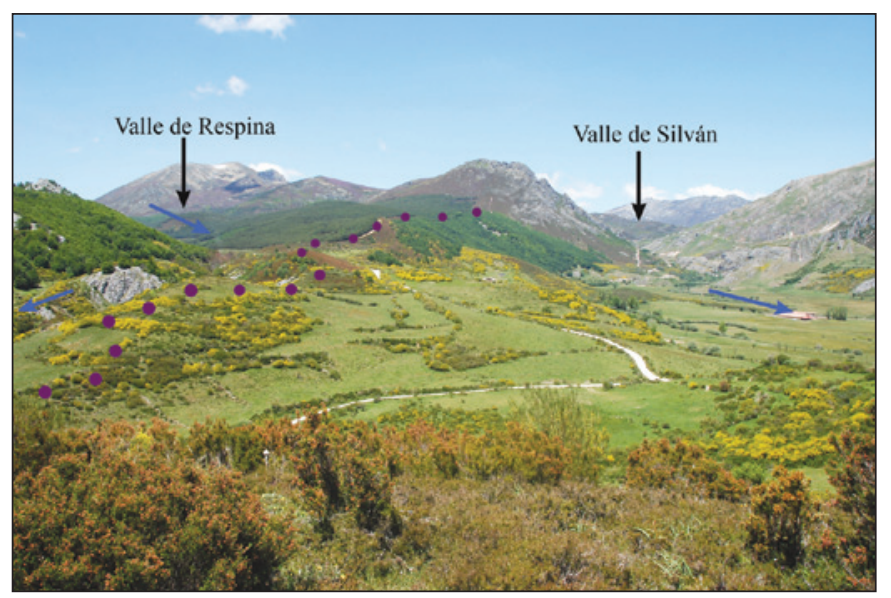

Figura 11. Vista de los valles de Silván y Respina. Destaca la potente acumulación morrénica de la margen derecha del valle de Silván (línea de puntos) que cierra la antigua salida del valle de Respina. Este gran conjunto morrénico, de más de $2.5 \mathrm{~km}$ de longitud, provocó el desvío del río Respina y la creación de un valle nuevo (Celorno). Nótese la menor altitud a la que se encuentra el valle de Silván respecto al de Respina, indicando una mayor potencia erosiva del primero sobre el segundo. Las flechas azules indican la dirección actual del avenamiento.

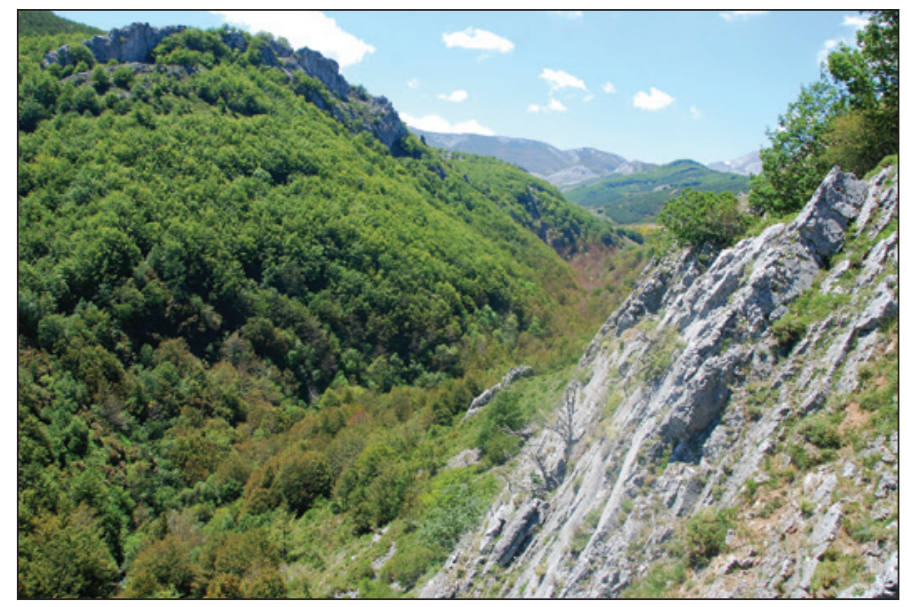

Figura 12. Vista del valle de Celorno, por donde discurre el río Respina. Las fuertes pendientes contrastan con la gran anchura de este mismo valle aguas arriba del arroyo de Rebueno, o del río Silván, que discurre paralelo inmediatamente más norte. 


\section{Conclusiones}

La morfología actual del valle de Respina es en gran parte derivada de la acción glaciar durante el Cuaternario. Así, en este valle aparecen prácticamente todos los rasgos característicos de una glaciación de tipo alpino, como el perfil transversal del valle en forma de artesa, valles afluentes suspendidos, morrenas que generan lagunas yuxtaglaciares o un magnífico conjunto de bloques erráticos repartidos por el valle. El complejo morrénico existente entre los valles de Silván y Respina es uno de los más potentes y mejor definidos de toda la Cordillera Cantábrica.

Esta diversidad de restos glaciares contrasta de forma significativa con su casi total ausencia en el valle de Rebueno, donde la acción glaciar se limitó a retocar la cabecera del valle principal. Por ello, el perfil, tanto longitudinal como transversal, es muy diferente al de Respina. La disponibilidad de sedimentos en las laderas es también inferior en Rebueno respecto a Respina, ya que los glaciares depositaron gran cantidad de materiales en este último valle. Estos sedimentos han sido posteriormente retrabajados por los barrancos laterales. Las diferencias morfológicas entre ambos valles ponen de manifiesto la importancia de la glaciación para entender el relieve actual de la Cordillera Cantábrica.

Pero lo que singulariza al valle de Respina es el reajuste de la red fluvial como consecuencia de la acción glaciar. En efecto, en la zona baja del valle, las morrenas laterales procedentes del valle de Silván impidieron el drenaje de las aguas del valle y las aguas de fusión glaciar contribuyeron a excavar el valle de Celorno en las lutitas y calizas del Grupo Lena. Este valle de origen más reciente presenta una morfología netamente diferente a los del entorno, con un perfil más angosto en forma de V.

Las observaciones realizadas sirven para mejorar el conocimiento existente sobre la morfología glaciar de la Cordillera Cantábrica en general, y de la cabecera del Porma en particular, donde se desarrolló uno de los mayores complejos glaciares del noroeste ibérico. La acción glaciar no solo dejó restos sedimentarios y erosivos muy notables, sino que contribuyó a establecer la red de drenaje preexistente, poniendo en evidencia la magnitud de la glaciación en estas montañas. En todo caso, aún es necesario profundizar notablemente en la influencia que la glaciación ha tenido en la configuración de la red de drenaje actual y en la dinámica fluvial cuaternaria.

\section{Referencias}

AEMET 2011. Atlas climático ibérico / Iberian climate atlas. Agencia Estatal de Meteorología, Ministerio de Medio Ambiente y Rural y Marino, Instituto de Meteorologia de Portugal, Madrid. Alonso Herrero, E. 2002. El glaciarismo en las cuencas altas de los ríos Esla y Porma. En: J.M. Redondo Vega, A. Gómez Villar, R.B. González Gutiérrez, P. Carrera Gómez (Coords.), El modelado de origen glaciar en las montañas leonesas, Universidad de León, León, pp. 235-248.

Alonso, M.V. 1998. El glaciarismo de la comarca de Laciana y alrededores. Zona occidental de la Cordillera Cantábrica. En: A. Gómez Ortiz, A. Pérez Alberti (eds.), Las huellas glaciares de las montañas españolas, Universidade de Santiago de Compostela, Santiago de Compostela, pp. 139-160. 
Alonso, J.L., Pulgar, J.A., García-Ramos, J.C., Barba, P. 1996. Tertiary basins and Alpine tectonics in the Cantabrian Mountains (NW Spain). In: P.F. Friend, C.J. Dabrio (eds.) Tertiary basins of Spain: Tectonics, Climate and Sea-Level Changes, Cambridge University Press, Cambridge, pp. 214-227.

Alonso, J.L., Marcos, A., Suárez, A. 2009. Paleogeographic inversion resulting from large out of sequence breaching thrusts: The León Fault (Cantabrian Zone, NW Iberia). A new picture of the external Variscan Thrust Belt in the Ibero-Armorican Arc. Geologica Acta 7 (4), 451-473. http://doi.org/10.1344/105.000001449.

Álvarez Marrón, J., Pérez Estaún,A. 1988. Thin skinned tectonics in the Ponga Region (Cantabrian Zone, NW Spain). Geologische Rundschau 77, 539-550. http://doi.org/10.1007/BF01832397.

Álvarez-Marrón, J., Pérez-Estaún, A., Aller, J., Heredia, N. 1990. Mapa Geológico Nacional a escala 1:50000. Hoja $n^{\circ} 79$ (Puebla de Lillo). I.T.G.E., Madrid.

Ballantyne, C.K. 2002. Paraglacial geomorphology. Quaternary Science Reviews 21, 1935-2017. http://doi.org/10.1016/S0277-3791(02)00005-7.

Benn, D.I., Evans, D.J.A. 2010. Glaciers and Glaciation, $2^{\text {nd }}$ Edition. Hodder Arnold Publication, $816 \mathrm{pp}$.

Benn, D.I., Gemmell, A.M.D. 1997. Calculating equilibrium-line altitudes of former glaciers by the balance ratio method: a new computer spread sheet. Glacial Geology and Geomorphology, http://ggg.qub.ac.uk/ggg/full/1997/tn011997/tn01.html.

Brardinoni, F., Hassan, M.A. 2006. Glacial erosion, evolution of river long profiles, and the organization of process domains in mountain drainage basins of coastal British Columbia. Journal of Geophysical Research 111, F01013. http://doi.org/10.1029/2005JF000358.

Brocklehurst, S.H., Whipple, K.X. 2006. Assessing the relative efficiency of fluvial and glacial erosion through simulation of fluvial landscapes. Geomorphology 75, 283-299. http://doi. org/10.1016/j.geomorph.2005.07.028.

Brook, M.S., Kirkbride, M.P., Brock, B.W. 2006. Quantified time scale for glacial valley crossprofile evolution in alpine mountains. Geology 34, 637-640. http://doi.org/10.1130/G22700.1.

Castañón Álvarez, J.C. 1989. Las formas de relieve de origen glaciar en los sectores central y oriental del macizo asturiano. Tesis Doctoral (inédita), Departamento de Geografía, Universidad de Oviedo, Oviedo, 787 pp.

Church. M., Ryder. J.M. 1972. Paraglacial sedimentation: consideration of fluvial processes conditioned by glaciation. Geological Society of America Bulletin 83, 3059-3072. http://doi. org/10.1130/0016-7606(1972)83[3059:PSACOF]2.0.CO;2.

Collins, B.D., Montgomery, D.R. 2011. The legacy of Pleistocene glaciation and the organization of lowland alluvial process domains in the Puget Sound region. Geomorphology 126 (1-2), 174-185. http://doi.org/10.1016/j.geomorph.2010.11.002.

Danis Álvarez, P.J. 2014. Geomorfología glaciar en la Cordillera Cantábrica: Valle de Respina. Trabajo de Fin de Grado. Departamento de Geografía y Geología, Universidad de León, 32 $\mathrm{pp}+1$ mapa geomorfológico.

De Prado, C. 1852. Note sur les blocs erratiques de la Chaine Cantabrique. Bulletin de la Societé Géologique de France 9, 171-175.

Fernández Martínez, E., Fuertes Gutiérrez, I., Alonso Herrero, E., Redondo Vega, J.M., Cortizo Álvarez, J., Gómez Villar, A., Santos González, J., Herrero Hernández, A., González Gutiérrez, R.B. 2009. Lugares de Interés Geológico. León. DVD. Fundación Patrimonio Natural, Junta de Castilla y León, 983 pp.

Frochoso, M., González-Pellejero, R., Allende, F. 2013. Pleistocene glacial morphology and timing of Last Glacial Cycle in Cantabrian Mountains (Northern Spain): new chronological data from the Asón Area. Open Geosciences 5 (1), 12-27. http://doi.org/10.2478/s13533012-0117-8. 
Frochoso Sánchez, M., Castañón Álvarez, J.C. 1998. El relieve glaciar de la Cordillera Cantábrica. En: A. Gómez Ortiz, A. Pérez Alberti (eds.) Las huellas glaciares de las montañas españolas, Universidade de Santiago de Compostela, Santiago de Compostela, pp. 65-137.

Furbish, D.J., Andrews, J.T. 1984. The use of hypsometry to indicate long-term stability and response of valley glaciers to changes in mass transfer. Journal of Glaciology 30 (104), 199-211.

García-Ruiz, J.M. 2015. Why geomorphology is a global science. Cuadernos de Investigación Geográfica 41 (1), 87-105. http://doi.org/10.18172/cig.2652.

García-Ruiz, J.M., Moreno, A., González Sampériz, P., Valero Garcés, B., Martí Bono, C. 2010. La cronología del último ciclo glaciar en las montañas del sur de Europa. Una revisión. Cuaternario y Geomorfología 24 (1-2), 35-46.

Gómez Villar, A. 1996. Conos aluviales en pequeñas cuencas torrenciales de montaña. Geoforma Ediciones, Logroño, $191 \mathrm{pp}$.

Gómez Villar, A., González Gutiérrez, R.B., Redondo Vega, J.M., Santos González, J. 2011. Distribución de glaciares rocosos relictos en la Cordillera Cantábrica. Cuadernos de Investigación Geográfica 37 (2), 49-80. http://doi.org/10.18172/cig.1256.

Gómez-Villar, A., Santos-González, J., González-Gutiérrez, R.B., Redondo-Vega, J.M. 2015. Glacial cirques in the southern side of the Cantabrian Mountains of southwestern Europe. Geografiska Annaler: Series A, Physical Geography 97 (4), 633-651. http://doi.org/10.1111/ geoa.12104.

González Gutiérrez, R.B. 2002. El relieve de los valles del Torío y Curureño (Montaña Cantábrica Leonesa). Universidad de León, León, 267 pp.

Hobley, D.E.J., Sinclair, H.D., Cowie, A. 2010. Processes, rates, and time scales of fluvial response in an ancient postglacial landscape of the northwest Indian Himalaya. Geological Society of America Bulletin 122 (9-10), 1569-1584. http://doi.org/10.1130/B30048.1.

Jalut, G., Turu i Michels, V., Deboubat, J.J., Otto, T., Ezquerra, J., Fontugne, M., Belet, J.M., Bonnet, L., García de Celis, A., Redondo-Vega, J.M., Vidal-Romaní, J.R., Santos, L., 2010. Palaeoenvironmental studies in NW Iberia (Cantabrian range): Vegetation history and synthetic approach of the last deglaciation phases in the western Mediterranean. Palaeogeography, Palaeocology, Palaeoclimatology 297 (2), 330-350. http://doi. org/10.1016/j.palaeo.2010.08.012.

Jiménez Sánchez, M. 1999. Geomorfología fluvial en la cabecera del río Nalón (Cordillera Cantábrica, Noroeste de España). Trabajos de Geología 21, 189-200.

Jiménez-Sánchez, J., Rodríguez-Rodríguez, J., García-Ruiz, J.M., Domínguez-Cuesta, M.J., Farias, P., Valero-Garcés, B., Moreno, A., Rico, M., Valcárcel, M. 2013. A review of glacial geomorphology and chronology in northern Spain: Timing and regional variability during the last glacial cycle. Geomorphology 196, 50-64. http://doi.org/10.1016/j. geomorph.2012.06.009.

Julivert, M. 1965. Sur la tectonique hercynienne à nappes de la Chaine Cantabrique (ètude geologique de la région à l'est du Bassin Central, Espagne). Bulletin de la Société Géologique de France 7 (4), 644-651.

Julivert, M., Fontboté, J.M., Ribeiro, A., Conde, L. 1972. Mapa Tectónico de la Península Ibérica y Baleares. I.G.M.E., 113 pp.

Lewis, C.J., McDonald, E.V., Sancho, C., Peña, J.L., Rhodes, E.J. 2009. Climatic implications of correlated Upper Pleistocene glacial and fluvial deposits on the Cinca and Gállego Rivers (NE Spain) based on OSL dating and soil stratigraphy. Global and Planetary Change 67, 141-152. http://doi.org/10.1016/j.gloplacha.2009.01.001.

Li, Y., Liu, G., Cui, Z. 2001. Glacial valley cross profile morphology, Tian Shan Mountains, China. Geomorphology 38, 153-166. http://doi.org/10.1016/S0169-555X(00)00078-7. 
Lotze, F. 1945. Zur Gliederung der Varisziden der Iberischen Meseta. Geotek. Frosch 6, 78-92.

MacGregor, K.R., Anderson, R.S., Waddington, E.D. 2009. Numerical modeling of glacial erosion and headwall processes in alpine valleys. Geomorphology 103, 189-204. http://doi. org/10.1016/j.geomorph.2008.04.022.

Montgomery, D.R. 2002. Valley formation by fluvial and glacial erosion. Geology 30, 1047-1050. http://doi.org/10.1130/0091-7613(2002)030<1047:VFBFAG>2.0.CO;2.

Moreno,A., Valero-Garcés, B.L., Jiménez-Sánchez, M., Domínguez-Cuesta, M.J., Mata, M.P., Navas, A., González-Sampériz, P., Stoll, H., Farias, P., Morellón, M., Corella, J.P., Rico, M. 2010. The last deglaciation in the Picos de Europa National Park (Cantabrian Mountains, northern Spain). Journal of Quaternary Science 25 (7), 1076-1091. http://doi.org/10.1002/jqs.1265.

Osmaston, H. 2005. Estimates of glacier equilibrium line altitudes by the Area x Altitude, the Area $x$ Altitude Balance Ratio and the Area $x$ Altitude Balance Index methods and their validation. Quaternary International 138-139, 22-31. http://doi.org/10.1016/j.quaint.2005.02.004.

Passmore, D.G., Waddington, C. 2009. Paraglacial adjustment of the fluvial system to Late Pleistocene deglaciation: the Milfield Basin, northern England. In: J. Knight, S. Harrison (eds.), Periglacial and Paraglacial processes and environments, Geological Society, Special Publication 320, 145-164. http://doi.org/10.1144/SP320.10.

Pérez Alberti, A., Valcárcel Díaz, M. 2001. Evolución morfogenética de las vertientes y del fondo de los valles durante el Tardiglaciar y el Postglaciar en el valle de Ancares (Serra dos Ancares, NW peninsular). En: F. Manero (Coord.), Espacio natural y dinámicas territoriales, Homenaje al Dr. D. Jesús García Fernández. Universidad de Valladolid y Universidad de Salamanca, pp. 115-125.

Rea, B.R. 2009. Defining modern day Area-Altitude Balance Ratios (AABRs) and their use in glacier-climate reconstructions. Quaternary Science Reviews 28 (3-4), 237-248. http://doi. org/10.1016/j.quascirev.2008.10.011.

Redondo Vega, J.M., Santos González, J., González Gutiérrez, R.B., Gómez Villar, A. 2013. Ejemplos de formas de relieve indicadoras de diferentes paleoclimas en la Cordillera Cantábrica. Polígonos 24, 163-181.

Redondo Vega, J.M., Alonso Herrero, E., García de Celis, A., Gómez Villar, A., González Gutiérrez, R.B., Santos González, J. 2014. Huellas glaciares a baja altitud en los valles cantábricos meridionales. En: J. Arnáez, P. González-Sampériz, T. Lasanta, B.L. ValeroGarcés (eds.), Geoecología, cambio ambiental y paisaje, Homenaje al Profesor José María García Ruiz. Instituto Pirenaico de Ecología (CSIC) y Universidad de La Rioja, Logroño, pp. 103-116.

Rodríguez Pérez, C. 1995. Estudio geomorfológico del puerto de San Isidro. Ería 36, 63-87.

Rodríguez Rodríguez, L. 2015. Quaternary glaciations in the central Cantabrian Mountains: new geomorphological and geochronological contributions. Tesis doctoral (inédita). Universidad de Oviedo.

Rodríguez-Rodríguez, L., Jiménez-Sánchez, M., Domínguez-Cuesta, M.J., Aranburu, A. 2015. Research history on glacial geomorphology and geochronology of the Cantabrian Mountains, North Iberia (43-42 $\left.\mathrm{N} / 7-2^{\circ} \mathrm{W}\right)$. Quaternary International 364, 6-21. http://doi.org/10.1016/j. quaint 2014.06.007.

Rodríguez-Rodríguez, L., Jiménez-Sánchez, M., Domínguez-Cuesta, M.J., Rinterknecht, V., Pallàs, R., Bourlès, D. 2016. Chronology of glaciations in the Cantabrian Mountains (NW Iberia) during the Last Glacial Cycle based on in situ-produced ${ }^{10} \mathrm{Be}$. Quaternary Science Reviews 138, 31-48. http://doi.org/10.1016/j.quascirev.2016.02.027.

Ruiz Fernández, J., Poblete Piedrabuena, M.A. 2011. Las terrazas fluviales del río Cares: aportaciones sedimentológicas y cronológicas (Picos de Europa, Asturias). Estudios Geográficos 270, 173-202. 
Ruiz-Fernández, J., Poblete-Piedrabuena, M.A., Serrano-Muela, M.P., Martí-Bono, C., GarcíaRuiz, J.M. 2009. Morphometry of glacial cirques in the Cantabrian Range (Northwest Spain). Zeitschrift für Geomorphologie 53 (1), 47-68. http://doi.org/10.1127/0372-8854/2009/00530047.

Ruiz-Fernández, J., Oliva, M., Cruces, A., Lopes, V., Freitas, M.C., Andrade, C., García-Hernández, C., López-Sáez, J.A., Geraldes, M. 2016. Environmental evolution in the Picos de Europa (Cantabrian Mountains, SW Europe) since the Last Glaciation. Quaternary Science Reviews 138, 87-104. http://doi.org/10.1016/j.quascirev.2016.03.002.

Saint-Saud, A.A. 1893. Les Picos de Europa (Monts Cantabriques). Etude Orographique. Annales Club Alpine Français, 38 pp.

Santos-González, J., Redondo-Vega, J.M., González-Gutiérrez, R.B., Gómez-Villar, A. 2013a. Applying the AABR method to reconstruct equilibrium-line altitudes from the last glacial maximum in the Cantabrian Mountains (SW Europe). Palaeogeography, Palaeoclimatology, Palaeoecology 387, 185-199. http://doi.org/10.1016/j.palaeo.2013.07.025.

Santos-González, J., Santos, J., González-Gutiérrez, R.B., Redondo-Vega, J.M., Gómez-Villar, A. 2013b. Till fabric and grain size analysis of glacial sequences in the Upper Sil River Basin, Cantabrian Mountains, NW Spain. Physical Geography 34 (6), 471-490. http://doi.org/10.1 080/02723646.2013.855989.

Serrano, E., Gómez-Lende, M., Pellitero, R., González-Trueba, J.J. 2015. Deglaciation in the Cantabrian Mountains: pattern and evolution. Cuadernos de Investigación Geográfica 41 (2), 389-408. http://doi.org/10.18172/cig.2716.

Serrano, E., González-Trueba, J.J., Pellitero, R., González-García, M., Gómez-Lende, M. 2013. Quaternary glacial evolution in the Central Cantabrian Mountains (Northern Spain). Geomorphology 196, 65-82. http://doi.org/10.1016/j.geomorph.2012.05.001.

Serrano-Cañadas, E., González-Trueba, J.J. 2004. El método AAR para la determinación de paleo-ELAs. Análisis metodológico y aplicación en el macizo de Valdecebollas (Cordillera Cantábrica). Cuadernos de Investigación Geográfica 30, 7-34. http://doi.org/10.18172. cig.1133.

Stickel, R. 1929. Observaciones de la morfología glaciar en el NO de España. Boletín de la Real Sociedad Española de Historia Natural 29, 297-313.

Turu, V., Peña, J.L. 2006. Las terrazas fluviales del sistema Segre-Valira (Andorra-La Seu d'Urgell Organyà, Pirineos Orientales): Parte I: relación con el glaciarismo y la tectónica activa. En: A. Pérez-Alberti, J. López Bedoya (eds.), Geomorfología y Territorio, Universidade Santiago de Compostela, IX Reunión Nacional de Geomorfología, pp. 113-128. 